\title{
QUANTITATIVE STRUCTURE-PHARMACOKINETICS MODELING OF THE UNBOUND CLEARANCE FOR NEUTRAL DRUGS
}

\author{
ZVETANKA ZHIVKOVA \\ Faculty of Pharmacy, Medical University, Sofia, Bulgaria \\ Email: zzhivkova@pharmfac.mu-sofia.bg
}

Received: 26 Dec 2017, Revised and Accepted: 05 Feb 2018

\begin{abstract}
Objective: Prediction of pharmacokinetic behaviour of new candidate drugs is an important step in drug design. Clearance is a key pharmacokinetic parameter, controlling drug exposure in the body. It depends on numerous factors and is frequently restricted by plasma protein binding. The study is focused on the development of quantitative structure-pharmacokinetic relationship (QSPkR) for the unbound clearance ( $\mathrm{CL}_{u}$ ) of neutral drugs.
\end{abstract}

Methods: The dataset consisted of 117 neutral drugs, divided into training set $(n=94)$ and external test set $(n=23)$. Chemical structures were encoded by 113 theoretical descriptors. Genetic algorithm and step-wise multiple linear regression were applied for model development. The model was evaluated by cross-validation in the training set and external test set.

Results: Significant, predictive and interpretable QSPkR model was developed with explained variance $\mathrm{r}^{2}=0.617$, cross-validated correlation coefficient $\mathrm{q}^{2}$ Loo-cV $=0.554$, external test set predictive coefficient $\mathrm{r}^{2}$ pred $=0.656$, and root mean square error in prediction RMSEP $=1.89$. The model was able to predict $\mathrm{CL}_{\mathrm{u}}$ for $56 \%$ of the drugs in the external test set within the 2 -fold error of experimental values.

Conclusion: The model reveals the main molecular features governing CLu of neutral drugs. $\mathrm{CL}_{\mathrm{u}}$ is favoured by lipophilicity, the presence of fused aromatic rings, ester groups, dihydropyridine moieties and nine-member ring systems, while polarity, molecular size and strong electron withdrawing atoms and groups as substituents in aromatic rings affect negatively $\mathrm{CL}$

Keywords: QSPkR, Clearance, Unbound clearance, In silico modelling, Prediction of ADME

(C) 2018 The Authors. Published by Innovare Academic Sciences Pvt Ltd. This is an open access article under the CC BY license (http://creativecommons.org/licenses/by/4.0/) DOI: http://dx.doi.org/10.22159/ijcpr.2018v10i2.25849

\section{INTRODUCTION}

Prediction of pharmacokinetic (PK) behaviour of new candidate drugs became a mandatory step in drug discovery process over the last two decades. Drug clearance (CL) is important pharmacokinetic (PK) parameter characterizing the ability of the body to eliminate the drug. It controls both half-lives, whence it is a major determinant of the dosage rate required for maintaining desired therapeutic concentration in multiple drug administration [1].

Several approaches have been developed for prediction of drug CLbased on in vivo data from preclinical species and allometric scaling, in vitro experiments, physiologically based, or in silico modelling. A brief review on the current state of methodology was published recently [2]. One of the most frequently applied techniques is quantitative structure-pharmacokinetics relationship modelling (QSPkR). QSPkR is a method of choice at very early stages of drug development as it can be based solely on easily computed molecular descriptors and allows predictions to be made even on virtual structures. It enables the screening of large databases of potential drug candidates and the choice of compounds with acceptable, if not ideal, PK properties.

Several reports have been published recently on QSPkR modeling of total plasma CL [3-9], as well as for renal CL [10-12]. It is difficult to compare their predictive performance because of the incomplete description of the model's algorithms and validation procedures and the different statistical metrics used. Some of the models were applicable only for ionized molecules [6], others showed different predictive accuracy for drugs of different ionization type [7, 10]. There was an agreement in three points:

- Prediction of total CL is rather a difficult task due to the involvement of multiple mechanisms in drug elimination. Most drugs are cleared via several pathways and their CL is determined by the rate and the extent of numerous processes such as uptake in liver, kidney and bile, metabolism, glomerular filtration, active secretion, reabsorption in kidney-each one with different structural requirements.

- CL may be restricted by the binding of drugs with plasma proteins, especially for drugs with low extraction ratio.

- Drugs follow elimination patterns depending on their ionization state. On average, acids have lower CL than neutral and bases. Analysis of a dataset of 754 compounds showed that $78 \%$ of the anionic drugs and $80 \%$ of zwitterions have low CL $(<4 \mathrm{ml} / \mathrm{min} / \mathrm{kg})$, and only $1-2 \%$-high CL $(>16 \mathrm{ml} / \mathrm{min} / \mathrm{kg})$. In contrast, most of the basic drugs have moderate (53\%) or high CL (18\%), and only $29 \%-$ low CL. Neutral drugs are in an intermediate position: $45 \%$ low-CL drugs, $39 \%$-moderate, and 16\%-high CL drugs [7]. Acidic drugs seem to be more often subjected to renal or biliary excretion, while basic drugs are cleared primarily by metabolism [13]. Different membrane transporters facilitate drug uptake into clearing organsorganic anion transporters for acids, and organic cation transportersfor bases [14]. Neutral drugs tend to show low renal CL ( $\left.C L_{R}\right)$, unless their $\log \mathrm{D}_{7.4}$ is negative. For drugs with $\log \mathrm{D}_{7.4}>0, \mathrm{CL}_{\mathrm{R}}$ decreases with the increase of lipophilicity due to tubular reabsorption [15]. Lipophilic drugs are expected to be cleared primarily by metabolism [16]. Considering CytP450 oxidation, anionic drugs are preferred substrates of CYP2C9, while most of the basic and neutral drugs are metabolized by CYP2C19 or CYP3A4 [13].

Given the above, development of separate QSPkR models according to the ionization type of the drugs seems reasonable as it could reveal the most significant structural features governing CL of drugs of different classes. The effect of plasma protein binding (PPB) could be avoided by the development of QSPkR for the unbound CL (CLu = $\mathrm{CL} / \mathrm{f}_{\mathrm{u}}$, where $\mathrm{f}_{\mathrm{u}}$ is the unbound fraction of the drug in plasma). $\mathrm{CL}_{u}$ is independent on PPB and is determined solely by molecular structure. Recently we published QSPkR models for CLu of anionic and cationic drugs $[17,18]$. The present study is focused on QSPkR modelling of $\mathrm{CL}$ for neutral molecules. 


\section{MATERIALS AND METHODS}

The dataset consisted of 117 neutral molecules with available data for $\mathrm{f}_{\mathrm{u}}$, extracted from the largest available database for key PK parameters following iv administration of drugs in human [19]. A drug was considered as neutral if the fraction ionized as an acid $\left(\mathrm{f}_{\mathrm{A}}\right)$ or as a base $\left(\mathrm{f}_{\mathrm{B}}\right)$ at $\mathrm{pH} 7.4$ didn't exceed $3 \%$. Drugs with $\mathrm{f}_{\mathrm{B}}>3 \%$ were classified as bases provided that $\mathrm{f}_{B}$ was considerably higher than $\mathrm{f}_{A}$. The fractions ionized at $\mathrm{pH} 7.4$ were calculated as previously described $[17,18]$. The mol-files of the drugs were derived from several public databasesDrug Bank, Chemical Book, or ChEBI [20-22].

The values of $C_{u}$ varied between 0.35 and 37,368 (mean $634 \pm$ 3,896 , median 17.68), and they were logarithmically transformed in order to achieve close to normal distribution. Thus, $\log \mathrm{CL}_{u}$ varied between-0.46 and 4.57 (mean 1.35, median 1.25). With respect to $\mathrm{CL}_{u}$ values, the drugs in the dataset could be classified into three groups:

- Low CL drugs: $\mathrm{CL}_{\mathrm{u}} \leq 4 \mathrm{ml} / \mathrm{min} / \mathrm{kg}(\mathrm{n}=29)$;

- Moderate CL drugs: $4<\mathrm{CLu}_{\mathrm{u}}<40 \mathrm{ml} / \mathrm{min} / \mathrm{kg}(\mathrm{n}=46)$

- High CL drugs: $\mathrm{CL}_{\mathrm{u}} \geq 40 \mathrm{ml} / \mathrm{min} / \mathrm{kg}(\mathrm{n}=42)$.

The whole dataset was divided into training and test sets. To this end, the molecules were arranged in an ascending order according to their $\mathrm{CL}_{u}$ values and one of every five drugs was allocated to the different subset. The first four subsets composed the training set for QSPkR model development $(n=94)$, and the fifth subset $(n=23)$ was used as a test set for external validation.

Chemical structures of the compounds were encoded by 113 molecular descriptors calculated with $\mathrm{ACD} / \log \mathrm{D}$ version 9.08 (Advanced Chemistry Development Inc., Ontario, Canada) and MDL QSAR version 2.2 (MDL Information Systems Inc, San Leandro, CA). Several types of descriptors were computed: physicochemical (logP, $\log \mathrm{D}_{7.4}, \mathrm{PSA}$, dipole moment), constitutional (number of atoms and groups of given type, rings, circles, hydrogen bond donors and acceptors, etc.); geometrical (volume, surface, ovality), electro topological state and connectivity indices, etc. The most significant descriptors were selected by genetic algorithm (GA) and stepwise linear regression (SWR). Both GA and SWR were implemented in the MDL QSAR package.

A number of successful QSPkR models were developed using multiple linear regression (MLR) and different combinations of descriptors. The goodness-of-fit was assessed using standard statistical metrics such as explained variance $\left(\mathrm{r}^{2}\right)$, root mean squared error (RMSE), Fisher criteria (F), etc. Drugs which $\log C L_{u}$ values were predicted with residuals not obeying normal distribution law were considered as outliers and were removed before building the final model.

Predictive ability of the developed QSPkR model was evaluated by internal leave-one-out cross-validation (LOO-CV) on the training set, and by the external test set not involved in any step of model development. The following statistical metrics were calculated: cross-validated coefficient for the training set $\left(\mathrm{q}^{2} \mathrm{Loo}-\mathrm{cv}\right)$, prediction coefficient for the external test set $\left(\mathrm{r}^{2}\right.$ pred $)$, mean fold error of prediction (MFEP), and root means square error of prediction (RMSEP), briefly described recently [2].

\section{RESULTS AND DISCUSSION}

The best QSPkR in terms of statistics given below:

$$
\begin{aligned}
& \log C L_{u}=0.237( \pm 0.031) * \log P+0.383( \pm 0.128) * \text { SaaaC_acnt }+0.179( \pm 0.038) * \text { Sdss } C_{-} a c n t+ \\
& +27.17( \pm 9.72) * x \operatorname{ch} 9-0.097( \pm 0.024) * Q_{s}-1.01( \pm 0.287) * H_{\min }+1.716
\end{aligned}
$$

$\mathrm{n}=90 \mathrm{r}^{2}=0.617 \mathrm{RMSE}=0.600 \mathrm{~F}=22.32$

Predictive ability of the model was assessed by LOO-CV on the training set and external validation on the independent test set. The $\mathrm{CV}$ squared correlation coefficient $\mathrm{q}^{2}$ Loo-cv $=0.554$ and external validation $\mathrm{r}^{2}$ pred $=0.656$ and RMSEP $=0.460$ meet the accepted criteria for good predictive QSAR models [23, 24]. The model was able to predict the $\mathrm{CL}_{u}$ of $56 \%$ of the drugs in the external test set within the 2 -fold error of experimental values. Six drugs were identified as outliers: four-from the training set, and two-for the test set. The plot of the observed $v$ s. predicted values of $\mathrm{CL}_{u}$ is shown in fig. 1 . The regression line is very close to the $\operatorname{line} \log \mathrm{CL}_{\mathrm{u}, \mathrm{obs}}=\log \mathrm{C} \mathrm{L}_{\mathrm{u}, \mathrm{pred}}$, which is a prove for a good predictive QSPkR model [24].

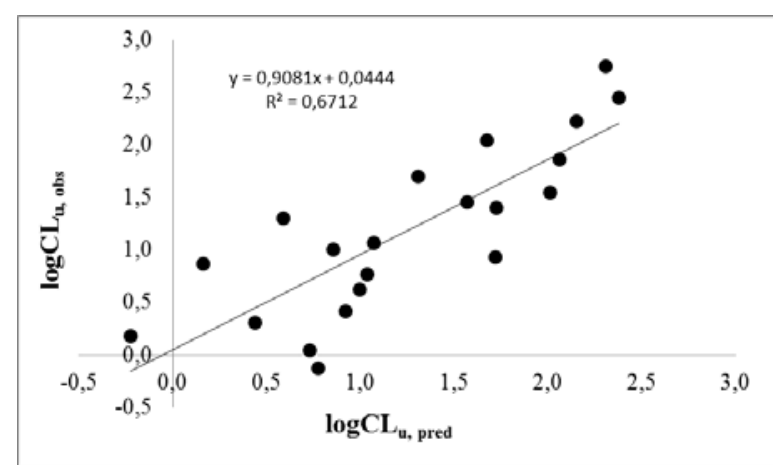

Fig. 1: A plot of observed vs predicted by the QSPkR model values of $\log C L u$

\section{The regression equation and regression coefficient are shown}

The descriptors in the developed QSPkR model have clear physical meaning and give insight to the main structural features governing $\mathrm{CL}_{u}$ of neutral drugs. Descriptors $\log \mathrm{P}, \mathrm{SaaaC}_{-}$acnt, Sdss C_acnt and xch4 contribute positively, while $\mathrm{Q}_{s}$ and $\mathrm{H}_{\min }$ disfavor $\mathrm{CL}_{u}$. Lipophilicity (expressed as $\log \mathrm{P}$ ) is the most important factor accounting for about $40 \%$ of the explained variance for $\log \mathrm{CL}_{\mathrm{u}}$. This is in agreement with the previous QSPkRs, and is quite reasonable as lipophilicity is a prerequisite for the main processes involved in drug elimination: uptake in the clearing organs and interactions at enzyme binding sites. Descriptor Saaa C_acntrepresents the number of aromatic C-atoms infused rings. This descriptor was found to affect positively PPBof both basic and neutral drugs $[25,26]$. The positive effect of Saaa $C_{-}$acnt on $\mathrm{CL}_{\mathrm{u}}$ may be due to the lowf $\mathrm{u}_{\mathrm{u}}$. Indeed, $60 \%$ of the drugs, containing aaaC atoms, have $\mathrm{f}_{\mathrm{u}}<0.1$. On the other hand, the presence of aromatic rings is a prerequisite for hydrophobic, van der Waals, $\mathrm{CH}-\pi$ and $\pi-\pi$ interactions in the binding sites of plasma proteins [27, 28], and the same interactions may be involved in the binding with transport proteins and metabolizing enzymes. Descriptor SdssC_acnt encodes the number of $\mathrm{C}$-atom connected with two simple and one double bond. It is presented in 78 molecules as $>\mathrm{C}=\mathrm{O}$ or $>\mathrm{C}=\mathrm{C}<$, and 13 of them are high-CL $\mathrm{C}_{\mathrm{u}}$ drugs. Among them are several dihydropyridine calcium channel blockers (isradipine, numodipine, nitrendipine, etc.) with common structural features: two $>\mathrm{C}=\mathrm{O}$ as a part of ester groups, and four $>\mathrm{C}=\mathrm{C}<$ in dihydropyridine moiety. They are extensively metabolized mainly via aromatization of the dihydropyridine moiety and oxidation of the two ester groups [29]. Fluticasone propionate also contains 4 dssC-atoms, one of which-part of fluoro methyl carbothioate group, which is metabolized extensively by liver CYP3A4 hydrolysis to inactive carboxylic acid metabolite [20]. Descriptor xch9 accounts for the presence, number and substituents in a 9-member ring system. The values of xch9 are higher for aromatic and non-saturated heterocycles. Aromatic structures are generally considered as more susceptible to oxidative metabolism. $\mathrm{Q}_{\mathrm{s}}$ represents molecular and group polarity index. It correlates significantly with molecular weight and surface and has high values for large molecules with many aromatic and non-aromatic rings. Majority of the large molecules in the dataset contain huge number hydrophilic atoms and groups like $\mathrm{C}=0,-\mathrm{OH},-\mathrm{NH}, \mathrm{NO}_{2}$, etc. and a large polar surface area (PSA), which is unfavorable for drug metabolism [30]. Descriptor $\mathrm{H}_{\text {min }}$ signifies the less polar $\mathrm{H}$-atom in the molecule. It has low values for $\mathrm{H}$-atoms in aliphatic chains and high values for $\mathrm{H}$-atoms in aromatic rings with electronegative substituents like $\mathrm{F}, \mathrm{Br}, \mathrm{Cl}, \mathrm{SO}_{2} \mathrm{R}$, etc. It is well known, that the presence of Cl-substituents increases metabolic stability by preventing aromatic hydroxylation and glucuronidation of phenols [31]. Involvement of substituents-strongly electron with-drawing atoms and groups such as $\mathrm{CF}_{3},-\mathrm{SO}_{2} \mathrm{NH}_{2}$, etc. is one of the recent 
strategies for improving metabolic stability by deactivation of aromatic rings again oxidative metabolism [32, 33].

Six drugs were identified as outliers from the QSPkR model. Four of them are very high CL drugs despite of the extensive PPB. They have extremely high $\mathrm{CL}_{u}$ values $(1,875-37,368 \mathrm{ml} / \mathrm{min} / \mathrm{kg})$, and were highly under-predicted. All they are subjected to extensive metabolism. Clevidipine butyrate is rapidly metabolized via hydrolysis by esterases in blood and extravascular tissues [34]. Maxipost undergoes $\mathrm{N}$-glucuronidation and $\mathrm{O}$-dealkylation to a metabolite, covalently bound to HSA [35]. Propofol metabolizes extensively in both liver and kidney, mainly by hydroxylation and glucuronidation [36, 37]. Estradiol undergoes extensive metabolism mainly by aromatic hydroxylation [38]. Decitabine is also very high CL drug (CL = $130 \mathrm{ml} / \mathrm{min} / \mathrm{kg}$ ), /however, it is essentially unbound in plasma. It undergoes hydrolysis and deamination mediated by cytidine deaminase in the liver, granulocytes, intestinal epithelium, and whole blood [39]. Its outlier behaviour may be due to the extremely low lipophilicity $(\log \mathrm{P}=-1$ 93). Meprobamate is the only drug which is over-predicted by the model. It has very low $\mathrm{CL}=0.6 \mathrm{ml} / \mathrm{min} / \mathrm{kg}$ and is completely unbound in plasma. It metabolizes to hydroxymeprobamate, meprobamateglucosyluronide and glucuronide conjugates, and $10-12 \%$ of a dose is excreted unchanged in urine [40]. Most probably, the low CL is due to the slow uptake in the liver as the drug is fairly hydrophilic $(\log \mathrm{P}=0.7)$.

\section{CONCLUSION}

A significant, validated and interpretable QSPkR model for the unbound plasma CL of neutral drugs is developed. The model is able to predict the $\mathrm{CL}_{\mathrm{u}}$ of $56 \%$ of the drugs in the independent external test set within the 2-fold error of experimental values. The descriptors in the model reveal molecular features, important for $\mathrm{CL}_{u} . \mathrm{CL}_{\mathrm{u}}$ is favored by lipophilicity, the presence of fused aromatic rings, ester groups, dihydropyridine moieties, and nine-member ring systems, while polarity, molecular size and strong electron withdrawing atoms and groups as substituents in aromatic rings affect negatively CL.

\section{AUTHORS CONTRIBUTIONS}

All the author have contributed equally

\section{CONFLICT OF INTERESTS}

\section{Declared none}

\section{REFERENCES}

1. Rowland M, Tozer TN. Elimination. In: Clinical Pharmacokinetics: concepts and application. $3^{\text {rd }}$ ed. Baltimore, Philadelphia, USA: Williams and Wilkins; 1995. p. 156-84.

2. Zhivkova Z. Application of QSPkR for prediction of key pharmacokinetic parameters. Lambert Academic Publishing; 2017.

3. Turner JV, Maddalena DJ, Cutler DJ. Pharmacokinetic parameter prediction from drug structure using artificial neural networks. Int J Pharm 2004;270:209-19.

4. Ng C, Xiao Y, Putnam W, Lum B, Tropsha A. Quantitative structure-pharmacokinetic parameters relationships (QSPkR) analysis of antimicrobial agents in human using simulated annealing k-nearest neighbor and partial least square analysis methods. J Pharm Sci 2004;93:2535-44.

5. Yap CW, Li ZR, Chen YZ. Quantitative structure-pharmacokinetic relationships for drug clearance by using statistical learning methods. J Mol Graph Model 2006;24:383-95.

6. $\mathrm{Yu}$ MJ. Predicting total clearance in humans from chemical structure. J Chem Inf Model 2010;50:1284-95.

7. Berellini G, Waters NJ, Lombardo F. In silico prediction of total human plasma clearance. J Chem Inf Model 2012;52:2069-78.

8. Gombar VK, Hall SD. Quantitative structure-activity relationship models of clinical pharmacokinetics: clearance and volume of distribution. J Chem Inf Model 2013;53:948-57.

9. Paine WS, Barton P, Bird J, Denton R, Menochet K, Smith A, et al. $A$ rapid computational filter for predicting the rate of human renal clearance. J Mol Graph Model 2010;29:529-37.

10. Lombardo F, Obach RS, Varma MV, Stringer R, Berellini G. Clearance mechanism assignment and total clearance prediction in human based upon in silico models. J Med Chem 2014;57:4392-405.

11. Manga NDJ, Duffy JC, Rowe PH, Cronin MTD. A hierarchical QSAR model for urinary excretion of drugs in humans as a predictive tool for biotransformation. QSAR Comb Sci 2003;22:263-73.

12. Doddareddy MR, Cho YS, Koh HY, Kim DH, Pae AN. In silico renal clearance model using classical volsurf approach. J Chem Inf Model 2006;46:1312-20.

13. Kusama M, Toshimoto K, Maeda K, Hirai Y, Imai S, Chiba K, et al. In silico classification of major clearance pathways of drugs with their physicochemical parameters. Drug Metab Dispos 2010;38:1362-70.

14. Pelis RM, Wright SH. Renal transport of organic anions and cations. Compr Physiol 2011;1:1795-835.

15. Smith DA, Allerton C, Kalgutkar A, Van de Waterbeemd H, Walker DK. Renal clearance. In: Pharmacokinetics and metabolism in drug design. $3^{\text {rd }}$ ed. Weinheim, Germany: WileyVCH Verlag GmbH and Co. KGaA; 2012. p. 103-10.

16. $\mathrm{Wu} \mathrm{CY}$, Benet LZ. Predicting drug disposition via application of BCS: transport/absorption/elimination interplay and development of a biopharmaceutics drug disposition classification system. Pharm Res 2005;22:11-23.

17. Zhivkova Z, Doytchinova I. Quantitative structure-clearance relationships of acidic drugs. Mol Pharmacol 2013;10:3758-68.

18. Zhivkova Z, Doytchinova I. Quantitative structurepharmacokinetic relationships for plasma clearance of basic drugs with consideration of the major elimination pathway. J Pharm Pharm Sci 2017;20:135-47.

19. Obach RS, Lombardo F, Waters NJ. Trend analysis of a database of intravenous pharmacokinetic parameters in humans for 670 drug compounds. Drug Metab Dispos 2008;36:1385-405.

20. http://www.drugbank.ca. [Last accessed on 20 Nov 2017]

21. http://chemicalbook.com. [Last accessed on 20 Nov 2017]

22. http://www.ebi.ac.uk. [Last accessed on 20 Nov 2017]

23. Roy K, Kar S, Das RN. Statistical methods in QSAR/QSPR. In: Roy K, Kar S, Das RN, editors. A primer on QSAR/QSPR modelling. Fundamental concepts. Heidelberg, New York, Dordrechs, London: Springer Cham; 2015. p. 37-59.

24. Alexander DLJ, Tropsha A, Winkler DA. Beware of R2: Simple, unambiguous assessment of the prediction accuracy of QSAR and QSPR models. J Chem Inf Model 2015;55:1316-22.

25. Zhivkova Z. Quantitative structure-pharmacokinetics relationships for plasma protein binding of basic drugs. J Pharm Pharm Sci 2017;20:349-59.

26. Zhivkova Z. Quantitative structure-pharmacokinetics relationship for plasma protein binding of neutral drugs, submitted for publication. J Pharm Pharm Sci 2017;20:349-59.

27. Hein KL, Kragh Hansen U, Morth JP, Jeppesen MD, Otzen D, Moeller JV, et al. Crystallographic analysis reveals a unique lidocaine binding site on human serum albumin. J Struct Biol 2010;171:353-60.

28. Nishi K, Ono T, Nakamura T, Fukunaga N, Izumi M, Watanabe $\mathrm{H}$, et al. Structural insights into differences in drug-binding selectivity between two forms of human alpha1-acid glycoprotein genetic variants, the $\mathrm{A}$ and $\mathrm{F} 1{ }^{*} \mathrm{~S}$ forms. J Biol Chem 2011;286:14427-34.

29. Al-Omarm MA. Nimodipine: drug metabolism and pharmacokinetic profile. Profiles of drug substances. Excipients Related Methodol 2005;31:371-5.

30. Smith DA, Allerton C, Kalgutkar A, Van de Waterbeemd H, Walker DK. Predicting human pharmacokinetics. In: Pharmacokinetics and metabolism in drug design. $3^{\text {rd }}$ ed. Weinheim, Germany: Wiley-VCH Verlag GmbH and Co. KGaA; 2012. p. 209-27.

31. Kerns EH, Di L. Drug-like properties: concepts, structure design and methods. $2^{\text {nd }}$ Ed. Elsevier: Amsterdam, Boston, Heidelberg, etc; 2016. p. 161-97.

32. Boehm HJ, Banner D, Bendels S, Kansy M, Kuhn B, Mueller K, et al. Fluorine in medicinal chemistry. Chem Bio Chem 2004;5:637-43.

33. Nassar AEF, Kamel AM, Clarimont C. Improving the decisionmaking process in the structural modification of drug candidates: enhancing metabolic stability. DDT 2004;9:1020-8. 
34. www.drugs.com/monograph/clevidipine-butyrate.html. [Last accessed on 20 Nov 2017]

35. Zhang D, Krishna R, Wang L, Zeng J, Mitroka J, Dai R, et al. Metabolism, pharmacokinetics, and protein covalent binding of radiolabeled MaxiPost (BMS-204352) in humans. Drug Metab Dispos 2005;33:83-93.

36. Hiraoka H, Yamamoto K, Miyoshi S, Morita T, Nakamura K, Kadoi Y, et al. Kidneys contribute to the extrahepatic clearance of propofol in humans, but not lungs and brain. Br J Clin Pharmacol 2005;60:176-82.
37. Favetta P, Degoute CS, Perdrix JP, Dufresne C, Boulieu R, Guitton J. Propofol metabolites in man following propofol induction and maintenance. Br J Anaesth 2002;88:653-8.

38. Tsuchiya Y, Nakajima M, Yokoi T. Cytochrome P450-mediated metabolism of estrogens and its regulation in human. Cancer Lett 2005;227:115-24.

39. Derissen EJB, Beijnen JH, Schellens JHM. Concise drug review: azacitadineand decitabine. Oncologist 2013;18:619-24.

40. American Society of Health-System Pharmacists; AHFS Drug Information. Bethesda MD; 2009. 